$48 \mid 2017$

L'art de mentir

\title{
Antoine Maire, La Mongolie entre dépendance et politiques développementalistes 1990-2016
}

\section{(2) OpenEdition \\ Journals}

Édition électronique

URL : https://journals.openedition.org/emscat/2959

DOI : $10.4000 /$ emscat.2959

ISSN : 2101-0013

Éditeur

Centre d'Etudes Mongoles \& Sibériennes / École Pratique des Hautes Études

Référence électronique

"Antoine Maire, La Mongolie entre dépendance et politiques développementalistes 1990-2016 », Études mongoles et sibériennes, centrasiatiques et tibétaines [En ligne], 48 | 2017, mis en ligne le 05 décembre 2017, consulté le 13 juillet 2021. URL : http://journals.openedition.org/emscat/2959; DOI : https://doi.org/10.4000/emscat.2959

Ce document a été généré automatiquement le 13 juillet 2021.

(c) Tous droits réservés 


\section{Antoine Maire, La Mongolie entre dépendance et politiques développementalistes 1990-2016}

\section{RÉFÉRENCE}

Thèse de science politique, mention relations internationales, soutenue au Centre de recherches internationales de Sciences Po, le 30 juin 2017 (835 pages). Membres du jury : B. Badie, F. Bafoil (directeur), A. Campi, B. Chavance (rapporteur), J.-F. Di Meglio, J. Legrand (rapporteur), T. Plaisant (tuteur)

1 Cette thèse cherche à évaluer la politique de développement mise en œuvre par les autorités mongoles entre 1990 et 2016. Elle s'articule autour de la question de recherche suivante: entre "malédiction des ressources", sinophobie et dépendance, comment la Mongolie démocratique et libérale fait-elle face au défi du développement économique pour préserver son indépendance et sa souveraineté et éviter d'être « transformée en une simple annexe de matières premières " pour la Chine?

2 À travers ce prisme, cette thèse ambitionne de rendre compte du processus de transition suivi par la Mongolie après 1990. L'objectif est de proposer une description du nouveau système politico-économique, donc des spécificités du capitalisme qui a vu le jour. Outre cette contribution aux études mongoles, cette thèse entend également permettre de mieux comprendre les défis auxquels sont confrontés les États riches en matières premières dans leur processus de développement. Enfin, cette thèse entend contribuer à une meilleure compréhension des mécanismes expliquant la divergence observée dans les processus de transitions politique et économique des pays postsocialistes. 


\section{Méthodologie de l'enquête}

3 La démonstration construite dans cette thèse s'est appuyée sur la construction d'un cadre théorique composite, nécessaire pour rendre compte de l'ensemble des spécificités du cas mongol. Cette thèse s'inscrit dans le cadre des approches dites de l'économie politique.

4 La confrontation des théories de la transition ${ }^{1}$, de la dépendance ${ }^{2}$ et de l'État développementaliste ${ }^{3}$ a conduit à l'élaboration de deux idéaux types : l'État dépendant et l'État développementaliste. Ceux-ci ont permis d'identifier cinq variables communes utilisées pour procéder à la description du cas mongol :

1. le rôle de l'État dans le développement économique ;

2. l'influence des grands conglomérats sur le processus de développement ;

3. la stratégie de développement ;

4. le financement du processus de développement et ;

5. l'alliance développementaliste, à savoir les relations que peuvent entretenir les principaux acteurs impliqués dans le processus de développement mongol.

5 La description du cas mongol permise par l'examen de ces variables a ensuite été confrontée à plusieurs hypothèses :

1. la Mongolie est un cas hybride, entre dépendance et développementalisme ;

2. le développement du secteur minier favorise une évolution du système politico-économique mongol vers le développementalisme ;

3. la démocratie favorise cette évolution tout en l'empêchant de devenir pleinement développementaliste ;

4. la démocratie constitue la clé de voute de la sécurité et de la nouvelle souveraineté mongole ;

5. le nouveau système politico-économique correspond aux caractéristiques intrinsèques de la société mongole.

\section{Structure de la thèse}

6 La problématique et le cadre théorique utilisés sont présentés dans l'introduction. La thèse examine chacune des variables identifiées afin de reconstruire le système politico-économique qui a vu le jour en Mongolie après la transition vers la démocratie et l'économie de marché.

7 Le premier chapitre est consacré à la question de l'État et au rôle qu'il joue dans le développement économique. Il explique pourquoi l'État est faible en Mongolie, quelles en sont les raisons, mais aussi ses conséquences pour le développement économique.

Le second chapitre est consacré à la question des groupes d'intérêts. Il présente leurs principales caractéristiques, le processus qui a conduit à leur formation et les liens qui les unissent au monde politique et à des partenaires étrangers. Il montre que ces groupes se sont pour le moment contentés de capter les différentes sources de rente que génère l'économie mongole, en particulier le secteur minier. Ces groupes sont néanmoins confrontés à un processus de transition. L'ensemble des positions de rentes étant distribuées, les grands groupes mongols, pour poursuivre leur processus de 
croissance, cherchent aujourd'hui à s'engager dans le développement de projets industriels.

9 Le troisième chapitre étudie la stratégie de développement adoptée par la Mongolie à travers son évolution depuis le début des années 1990 et son influence sur le processus de développement. Malgré leur nombre important, les textes d'orientation n'ont qu'une faible influence sur le processus de développement, l'État mongol ne disposant d'aucun outil d'intervention dans l'économie. Une évolution est cependant notable ces dernières années, l'État tendant à se doter de nouveaux outils d'intervention, qu'ils soient réglementaires ou financiers, et qui renforcent sa capacité à orienter le processus de développement.

10 Le quatrième chapitre étudie le financement du processus de développement mongol. Il présente les sources de financement sur lesquelles s'appuient les autorités : l'épargne domestique, la rente minière, les investissements étrangers, et les dons et prêts internationaux. Il apparaît que les autorités mongoles recourent de manière indiscriminée à l'ensemble de ces sources de financement. Ce chapitre met également en évidence les tâtonnements et le processus d'apprentissage qu'ont dû suivre et que suivent encore les autorités. Ce processus d'apprentissage concerne à la fois la gestion de la rente minière, le contrôle des investissements étrangers ou encore la redistribution de l'argent collecté dans le cadre de l'émission d'obligations sur les marchés étrangers. L'importance du facteur politique dans la prise de décision limite la capacité des autorités mongoles à utiliser l'argent issu de ces différentes sources de financement dans le cadre d'un processus de développement.

11 Le cinquième et dernier chapitre examine la façon dont interagissent l'ensemble des acteurs évoqués précédemment autour du projet minier de Tavan Tolgoi (province Ömnögov') et questionne la possibilité de voir émerger en Mongolie une «alliance développementaliste ». Ce dernier chapitre réintroduit les acteurs étrangers, notamment chinois et russe et démontre que la situation géographique dans laquelle se trouve la Mongolie, notamment son enclavement géographique et les convoitises stratégiques qu'elle peut susciter, se traduit par une importation des tensions extérieures par les acteurs domestiques. Dans ce cadre, les divisions internes et externes compliquent dès lors l'apparition d'une alliance développementaliste.

12 La conclusion de cette thèse réunit les enseignements collectés et dresse le portrait du système politico-économique qui a vu le jour en Mongolie après la transition. Un certain nombre de questions et de points restent cependant en suspens, qui sont autant de nouvelles pistes de recherche.

\section{Principaux résultats}

13 Cette thèse a permis de décrire les caractéristiques du nouveau système politicoéconomique qui a vu le jour en Mongolie après la transition démocratique. Ce système emprunte à la fois au modèle de la théorie de la dépendance et à celui de l'État développementaliste. Le cas mongol est donc un cas hybride. Une évolution apparaît néanmoins notable. Après avoir opté pour une politique économique d'inspiration néolibérale, la politique a progressivement évolué sous l'influence du développement du secteur minier pour devenir plus interventionniste et plus dirigiste. Le développement du secteur minier impose en effet à l'État de réguler ce secteur pour s'assurer que le développement de cette activité profite autant que possible au pays. La rente générée 
par cette activité ouvre également de nouvelles possibilités d'action qui permettent à l'État de s'impliquer concrètement dans le processus de développement économique et de tenter de mettre en œuvre une stratégie d'industrialisation. Cette évolution vers le développementalisme est néanmoins limitée par le fonctionnement de la démocratie mongole. Les alternances politiques dont le pays est coutumier fragilisent l'État, réduisent sa capacité d'intervention, et surtout, en limitent la capacité à agir dans un temps qui n'est pas uniquement le temps politique. Cette faiblesse de l'État constitue dès lors un des obstacles majeurs à sa transformation en un état réellement développementaliste. Cependant, il apparaît également que la démocratie constitue un facteur essentiel de la nouvelle souveraineté conquise par les Mongols après 1990. Du fait de l'incertitude inhérente à ce mode de prise de décision, ce système génère des marges de manœuvre pour les autorités et les protège contre les pressions auxquelles elles pourraient être exposées, notamment celles liées à leur enclavement géographique. Outre cette situation, cette thèse a également démontré que le système politique et économique de la Mongolie démocratique correspond aux caractéristiques intrinsèques de la société mongole, notamment marquée par le nomadisme pastoral.

\section{BIBLIOGRAPHIE}

Amsden, A. H. 1989 Asia's Next Giant. South Korea and Late Industrialization (Oxford, Oxford University Press).

Bafoil, F. 2006 Europe centrale et orientale. Mondialisation, européanisation et changement social (Paris, Presses de Sciences Po).

Bunce, V. 1999 The political economy of postsocialism, Slavic Review 58(4), pp. 756-793.

Cardoso, F. H. \& E. Faletto 1978 Dépendance et développement en Amérique Latine (Paris, Presses universitaires de France).

Dobry, M. (dir.) 2000 Les transitions démocratiques regards sur l'état de la "transitologie ", numéro spécial, Revue Française de Science Politique 50(4-5).

Dufy, C. \& C. Thiriot 2013 Les apories de la transitologie : quelques pistes de recherche à la lumière d'exemples africains et post-soviétiques, Revue internationale de politique comparée 20(3), pp. 19-40.

Evans, P. 1979 Dependent Development. The Alliance of Multinational, State, and Local Capital in Brazil (Princeton, Princeton University Press).

Frank, A. G. 1967 Capitalisme et sous-développement en Amérique latine (Paris, Maspero).

Johnson, C. 1982 MITI and the Japanese Miracle. The Growth of Industrial Policy, 1925-1975 (Stanford, Stanford University Press).

Linz J. J. \& A. Stepan 1996 Problems of Democratic Transition and Consolidation (Baltimore, John Hopkins University Press). 
O'Donnell, G., Schmitter, P. \& L. Whitehead, Laurence (eds) 1986, Transitions From Authoritarian Rule (Baltimore, John Hopkins University Press).

Packenham, R. A. 1992 The Dependency Movement. Scholarship and Politics in Development Studies (Cambridge, MA, Harvard University Press).

Poulantzas, N. 1974 Les classes sociales dans le capitalisme aujourd'hui (Paris, Seuil).

Woo-Cumings, M. (ed.) 1999 The Developmental State (Ithaca, Cornell University Press).

\section{NOTES}

1. Voir notamment O’Donnell et al.1986; Linz \& Stepan 1996; Bunce 1999; Dobry 2000; Bafoil 2006 ; Dufy \& Thiriot 2013.

2. Voir notamment Frank 1967 ; Poulantzas 1974 ; Cardoso \& Faletto 1978 ; Packenham 1992.

3. Voir notamment Evans 1979 ; Johnson 1982 ; Amsden 1989 ; Woo-Cumings 1999. 